\title{
RINGS WITH NONDISCRETE IDEAL TOPOLOGIES ${ }^{1}$
}

\section{HOCHSTER}

0. Introduction. A proper topology on a ring $A$ (all rings are supposed commutative, with unit) is simply a nondiscrete Hausdorff ring topology on $A$. A proper topology will be called an ideal topology if there is a neighborhood basis (equivalently, subbasis) for 0 consisting of ideals. We shall obtain a satisfactory ring-theoretic characterization of those rings which have ideal topologies. In the Noetherian case we get the result that a Noetherian ring has no ideal topology iff it is Artin. We also study what happens in case of localization and the taking of direct products. Recently, J. O. Kiltinen has shown $[1$, p. 69] and [2] that every infinite field has a proper topology. Using this fact and our results on ideal topologies we show that every infinite Noetherian ring has a proper topology. We note that most of the ring topologies defined in commutative algebra are ideal topologies, including the usual valuation topologies.

1. The characterization. Let $A$ be a set, $J \subset A$, and $\Sigma$ a family of subsets of $A$. We say that $\Sigma$ has the FIP/J if no finite intersection of sets in $\Sigma$ is contained in $J$. Now, a ring topology is determined once a subbasis for the neighborhoods of 0 is known, and it is a standard fact that a family $\Sigma$ of ideals of a ring $A$ is a neighborhood subbasis generating a proper topology iff $\Sigma$ has the $F I P /(0)$ and $\bigcap_{I \in \Sigma} I=(0)$. In this case, the closure of $\Sigma$ under finite intersection is a neighborhood basis for 0 .

A trivial observation, apparently more curious than useful, is that a ring $A$ has no ideal topology iff $A-\{0\}$ is quasi-compact in the topology having as a subbasis for its closed sets the family of sets $\{I-\{0\}: I$ an ideal of $A\}$.

We now prepare to give our characterization of rings with no ideal topology. We write $M(A)$ for the set of maximal ideals of $A$ and $M_{0}(A)$ for $\left\{m \in M(A): \operatorname{Ann}_{A} m \neq(0)\right\}$. We define four properties of rings $A$ which we shall need to consider and then proceed to the statement of our result.

$\mathrm{P} 1 . M_{0}(A)$ is finite.

$\mathrm{P} 1^{\circ}$. There is a finite subset $F$ of $M(A)$ whose union contains all the zero divisors in $A$.

P2. For each $m \in M(A), \mathrm{Ann}_{A} m$ is finitely generated.

Received by the editors June 13, 1968

1 Research partially supported by NSF grant GP-8496. 
P3. For each $a \in A-\{0\},(a) \cap\left(\bigcup_{m \in M(\Delta)} A_{n n} m\right) \neq\{0\}$, where $(a)=a A$, the principal ideal generated by $a$.

THEOREM 1. The following conditions on a ring $A$ are equivalent.

(i) $A$ has no ideal topology.

(ii) $A$ has properties $\mathrm{P} 1, \mathrm{P} 2$, and $\mathrm{P} 3$.

(iii) $A$ has properties $\mathrm{P} 1^{\circ}, \mathrm{P} 2$, and $\mathrm{P} 3$.

Proof. Note that $P 1^{\circ} \Rightarrow \mathrm{P} 1$ without the presence of (ii) and (iii). For $\operatorname{Ann}_{A} I \neq(0) \Rightarrow I$ consists entirely of zero divisors and hence $I \subset \bigcup_{m \in F} m \Rightarrow I \subset m$ for some $m \in F$, since $F$ is finite and the ideals $m$ are prime. But then $I$ maximal and $A_{A n n_{A}} I \neq(0) \Rightarrow I \subset m$, some $m \in F \Rightarrow I=m$. Thus, (iii) $\Rightarrow$ (ii). It remains to show that (ii) $\Rightarrow(\mathrm{i})$ and (i) $\Rightarrow$ (iii).

Assume (ii), and let $\Sigma$ be a family of ideals of $A$ closed under finite intersection but such that $(0) \notin \Sigma$. We shall prove that $\bigcap_{I \in \Sigma} I \neq(0)$. Let $F=\left\{m_{1}, \cdots, m_{n}\right\}=M_{0}(A)$ ( $F$ is finite by $\left.\mathrm{P} 1\right)$. For each $i$, $\mathrm{Ann}_{A} m_{i}$ can be regarded as a vector space $V_{i}$ over $k_{i}=A / m_{i}$, and its vector subspaces as a vector space over $k_{i}$ are identical (as sets) with its submodules as an $A$-module, i.e. with its subideals. By $\mathrm{P} 2$, each $V_{i}$ is finite dimensional. For each $i$, let $d_{i}$ be the least of the dimensions (over $k_{i}$ ) of the subspaces $I \cap V_{i}, I \in \Sigma$, and choose $I_{i}$ in $\Sigma$ such that the dimension of $I_{i} \cap V_{i}$ over $k_{i}$ is $d_{i}$. Let $I_{0}=\bigcap_{i} I_{i}$. Then $I_{0}$ is in $\Sigma$ and for each $i$ the dimension of $I_{0} \cap V_{i}$ is less than or equal to $d_{i}$, since $I_{0} \subset I_{i}$. The other inequality follows from the choice of $d_{i}$ and we have that the dimension of $I_{0} \cap V_{i}$ over $k_{i}=d_{i}$ for each $i$. We next show that for at least one $i, d_{i} \neq 0$. As a matter of fact, $d_{i}=0$ iff $I_{0} \cap V_{i}$ $=(0)$ and if all the $d_{i}$ were zero, we would have that $\{0\}=U_{i}\left(I_{0} \cap V_{i}\right)$ $=U_{m \in M(A)}\left(I_{0} \cap \mathrm{Ann}_{A} m\right)$ (recall that $\mathrm{Ann}_{A} m=(0)$ if $m$ is not in $F$ ) $=I_{0} \cap\left(\bigcup_{m \in M(A)} A_{n n_{A}} m\right)$, contradicting P3.

Suppose, say, $d_{1} \neq 0$. Then for all $I$ in $\Sigma, I_{0} \cap V_{1} \subset I$. For if not, then $\left(I \cap I_{0}\right) \cap V_{1}$ is a proper subspace of $I_{0} \cap V_{1}$ and hence of lower dimension than $d_{1}$, a contradiction. But then $\bigcap_{I \in \Sigma} I \supset I_{0} \cap V_{1} \neq(0)$ (since $\left.d_{1} \neq 0\right)$, and we have that $\bigcap_{I \in \Sigma} I \neq(0)$, as required.

(i) $\Rightarrow$ (iii) remains. Assume that $A$ has no ideal topology. We must deduce $\mathrm{P} 1^{\circ}, \mathrm{P} 2$, and $\mathrm{P} 3$. To establish $\mathrm{P} 1^{\circ}$, consider the family of ideals $\Sigma=\left\{I_{m}: m \in M(A)\right\}$, where for each $m, I_{m}=\left\{a \in A: \operatorname{Ann}_{A} a \not m\right\}$. Of course, $I_{m}=\operatorname{Ker}\left(A \rightarrow A_{m}\right)$, the kernel of the canonical homomorphism from $A$ to its localization at $m$. Let $J=\bigcap_{I \in \Sigma} I . J=(0)$, for if $0 \neq a \in J$, we would have $\mathrm{Ann}_{A} a \nsubseteq m$ for every maximal ideal $m$. Now, since $A$ has no ideal topology it follows that for some finite subset $F \subset M(A), \bigcap_{m \in F} I_{m}=(0)$. This means that if $\mathrm{Ann}_{A} a \nsubseteq m$ for each $m$ in $F$, then $a=0$, i.e. $a \neq 0 \Rightarrow \operatorname{Ann}_{A} a \subset m$ for some $m$ in $F$. Thus, if $b$ is a 
zero divisor, then $b \in \mathrm{Ann}_{A} a$ for some $a \neq 0$, and we get that $b \in m$ for some $m$ in $F$. This proves that $\mathrm{P} 1^{\circ}$ holds.

We next show that P2 must hold. Suppose that $\mathrm{Ann}_{A} m$ is not finitely generated. Then it is infinite dimensional when regarded as a vector space $V$ over $k=A / m$. We can then choose a countable infinite decreasing sequence of subspaces $V_{1}, V_{2}, \ldots$ of $V$ such that $\bigcap_{n} V_{n}=(0)$. (E.g. let $v_{1}, v_{2}, \cdots$ be a countable infinite sequence of linearly independent elements of $V$ and let $V_{n}$ be the subspace of $V$ spanned by the $v_{i}, i \geqq n$.) But these $V_{n}$ will be nonzero subideals of $\mathrm{Ann}_{A} m$, and will constitute a neighborhood basis for 0 of a proper ideal topology.

$\mathrm{P} 3$ only remains. Let $a \neq 0$ be given. By Zorn's lemma, the family of ideals $\{(a)\}$ can be extended to a maximal family $\Sigma$ having the $F I P /(0)$. Since $A$ has no ideal topology, we can conclude that $J$ $=\bigcap_{I \in \Sigma} I \neq(0)$. Then clearly, $J \subset(a)$ and $\Sigma$ is merely the set of all ideals of $A$ containing $J$. Moreover $J$ cannot have a proper nonzero subideal $J^{\prime}$ or the set of all ideals of $A$ containing $J^{\prime}$ would properly extend $\Sigma$. It follows that $J$ is a simple $A$-module, hence isomorphic as an $A$-module with $A / m$ for some $m$ in $M(A)$. For any $b \neq 0$ in $J$, we have $b \in(a)$ and $b \in \mathrm{Ann}_{A} m$, and therefore $(a) \cap\left(\bigcup_{m \in M(A)} \mathrm{Ann}_{A} m\right)$ $\neq\{0\}$. This completes the proof.

\section{Products.}

ThEOREM 2. A finite product of rings has no proper topology [resp. no ideal topology] iff each factor has no proper topology [resp. no ideal topology]. An infinite product of rings or, more generally, a ring $A$ such that Spec $A$ has infinitely many components always has an ideal topology.

Proof. We give the proof of the first statement for the case of proper topologies; we then indicate how it needs to be modified for the ideal case. By a trivial induction, we need only treat the case of two rings, $A_{1}, A_{2}$. If $\Sigma$ is a neighborhood basis for 0 for a proper topology on $A_{1}$ then $\Sigma \times 0=\{I \times 0: I \in \Sigma\}$ is such for $A_{1} \times A_{2}$. What remains to be shown is that if neither $A_{1}$ nor $A_{2}$ has a proper topology then $A_{1} \times A_{2}$ has none as well. Suppose, to the contrary, that $\Sigma$ is a neighborhood basis for 0 , closed under finite intersection, generating a proper topology for $A_{1} \times A_{2}$. For each $I$ in $\Sigma$ let $I_{1}=\left\{a_{1} \in A_{1}:\left(a_{1}, 0\right) \in I\right\}$. It is easy to see that $\Sigma_{1}=\left\{I_{1}: I \in \Sigma\right\}$ will be a neighborhood basis for 0 generating a proper topology on $A_{1}$ unless $\Sigma_{1}$ fails to have the $F I P /(0)$, i.e. fails to contain (0). It follows that for all sufficiently small $I, I_{1}=(0)$. Restricting attention to those elements of $\Sigma$ which are 
contained in one such $I$, we may assume without loss of generality that $I_{1}=(0)$ for every $I$ in $\Sigma$. Now let $p: A_{1} \times A_{2} \rightarrow A_{2}$ be the product projection and consider $p \Sigma=\{p(I): I \in \Sigma\}$. It is again easy to see that $p \Sigma$ will be a neighborhood basis for 0 generating a proper topology on $A_{2}$ unless the sets in $p \Sigma$ have nonzero intersection. Hence, we can choose $0 \neq b \in A_{2}$ such that for all $I$ in $\Sigma$ there exists $a(I)$ in $A_{1}$ such that $(a(I), b) \in I$. Fix $I, J$ in $\Sigma$ such that $J-J \subset I$. Then $0 \neq(a(J), b)$ is in the intersection of all the sets in $\Sigma$, a contradiction. For otherwise $(a(J), b) \notin J^{\prime}$ for some $J^{\prime} \subset J$, and in particular we then have $(a(J), b)$ $\neq\left(a\left(J^{\prime}\right), b\right)$. The difference of these two, $\left(a(J)-a\left(J^{\prime}\right), 0\right) \in J-J^{\prime}$ $\subset J-J \subset I$, which yields that $a(J)-a\left(J^{\prime}\right) \in I_{1}=(0)$, a contradiction. This completes the proof of the first statement in the case of proper topologies. For the ideal case, it is only necessary to observe in addition that if $\Sigma$ is a family of ideals, so is $\Sigma \times 0$, and that if $\Sigma$ is a family of ideals, so are $\Sigma_{1}$ and $p \Sigma$.

We now consider the second statement of the theorem. For any ring $A$, let $\#(A)$ be the cardinality of $M_{0}(A)$. Note that $\#\left(A_{1} \times A_{2}\right)$ $=\#\left(A_{1}\right)+\#\left(A_{2}\right)$ (no finiteness conditions). In fact, under the usual correspondence between Spec $A_{1} \times A_{2}$ and the topological union of Spec $A_{1}$ and Spec $A_{2}$ it is easy to see that $M_{0}\left(A_{1} \times A_{2}\right)$ corresponds to $M_{0}\left(A_{1}\right) \cup M_{0}\left(A_{2}\right)$. We also observe that if $A$ has no ideal topology, then $1 \leqq \#(A)<\infty$. The first inequality follows from $\mathrm{P} 3$, the second from $\mathrm{P} 1$. Now suppose that for a certain $\operatorname{ring} A, \operatorname{Spec} A$ has infinitely many components. Then $A$ has arbitrarily long (finite) direct product decompositions. Assume that $A$ has no ideal topology. Let $A=A_{1}$ $\times \cdots \times A_{n}$ be a direct product decomposition of $A$ with $n$ factors. Each $A_{i}$ has no ideal topology (since $A$ does not) and hence $\#\left(A_{i}\right) \geqq 1$ for each $i$. But then $\#(A)=\#\left(A_{1}\right)+\cdots+\#\left(A_{n}\right) \geqq n$, and since this holds for every $n$ we have a contradiction of $\#(A)<\infty$.

3. Localization. Let $A$ be a ring and let $F \subset M(A)$ be a finite set such that every zero divisor is in one of the ideals in $F$. Let $S$ be the complement of $\bigcup_{m \in F} m$ in $A . S$ is a multiplicative system consisting entirely of nonzero divisors. Hence, the canonical homomorphism $A \rightarrow S^{-1} A=B$ is injective.

Theorem 3. If one of $A, B$ above has no ideal topology, then the other also has no ideal topology.

Proof. We first observe that $m \mapsto m B$ is a bijection from $F$ onto $M(B)$ and that $M_{0}(B)$ is the image of $M_{0}(A)$. Thus, $\mathrm{P} 1^{\circ}$ and $\mathrm{P} 1$ hold for both $A$ and $B$. We next observe that $\mathrm{Ann}_{B} m B=\mathrm{Ann}_{A} m$ for each $m$ in $M_{0}(A)$. It is clear that $\mathrm{Ann}_{B} m B=\left(\mathrm{Ann}_{A} m\right) B$, and we also know 
that we can already multiply elements of $A n_{A} m$ by elements $1 / s$, $s \in S$, by multiplying instead by a suitable element of $A$, because $s \notin m \Rightarrow$ the image of $s$ in $A / m$ is invertible and the structure of $A n_{A} m$ as an $A$-module is essentially the same as its structure as an $(A / m)$ module. In particular, $A n_{B} m B$ is finitely generated over $B$ iff $A_{n n} m$ is finitely generated over $A$. Hence, $\mathrm{P} 2$ holds for $A$ iff it holds for $B$. Also, $U_{m \in M(A)} A_{n n_{A}} m=U_{m^{\prime} \in M(B)} A_{n n_{B}} m^{\prime}$, and each nonzero element of $B$ has a nonzero multiple in $A$, and thus P3 holds for $A$ iff it holds for $B$. The proof is complete.

4. The Noetherian case. Henceforth all rings are assumed Noetherian. It is clear that $A$ Artin $\Rightarrow A$ has no ideal topology. Now suppose that $A$ is semilocal but has no ideal topology. Then if $J$ is the Jacobson radical of $A$, we have $\bigcap_{n} J^{n}=(0) \Rightarrow$ for some $n, J^{n}=(0) \Rightarrow A$ Artin. This converse holds, however, even without the assumption that $A$ is semilocal.

Theorem 4. If $A$ is Noetherian, $A$ has no ideal topology iff $A$ is Artin.

Proof. We need only show that if $A$ has no ideal topology then it is semilocal. Choose a finite set $F_{1}=\left\{m_{1}, \cdots, m_{n}\right\} \subset M(A)$ such that every zero divisor in $A$ is in one of the $m_{i}$. We shall show that $F_{1}$ $=M(A)$. For suppose not; choose $m \in M(A)-F_{1}$ and let $F=\{m\} \cup F_{1}$. Applying the results of the preceding section with this $A$ and $F$, we find that $B$ is a semilocal ring with no ideal topology, hence, Artin. Now $m$ does not consist entirely of zero divisors (since $m \nsubseteq U_{i} m_{i}$ ). Let $a$ be a nonzero divisor in $m$. Then $a$ is also a nonzero divisor in $m B$. But $a^{n} B=a^{n+1} B$ for some $n$ because $B$ is Artin, and thus $a^{n}$ $=a^{n+1} b$ for some $b$ in $B$. But then $a^{n}(1-a b)=0$, and since $a$ is not a zero divisor, it follows that $1-a b=0$, i.e. that $a$ is a unit, contradicting $a \in m B$.

Theorem 5. Every infinite Notherian ring $A$ has a proper topology.

Proof. We may assume that $A$ is Artin. Then $A$ is a finite product of Artin local rings, i.e. of local rings in which the maximal ideal is nilpotent (see [3, pp. 25-26 and 4, pp. 203-208] for the relevant facts). At least one factor must be infinite, and since it is enough for one factor to have a proper topology, we may assume without loss of generality that $(A, m)$ is local, infinite, and that $m^{n}=(0)$ while $m^{n-1} \neq(0), n \geqq 1$. If $A / m=k$ were a finite field, then each of $A / m$, $m / m^{2}, \cdots, m^{n-1} / m^{n}=m^{n-1}$ would be a finite dimensional vector space over a finite field, and it would follow that $A$ must be finite. 
Hence, we can assume that $k$ is infinite. From the results of Kiltinen $[1$, p. 69], we know that $k$ has a proper topology. Let $\Sigma$ be a neighborhood basis for 0 in some proper topology for $k$. Now choose $a \in m^{n-1}$ $-\{0\}$. Then $a \in A_{A n n_{A} m}$ and hence $k \cong(a)$ as an $A$-module. Let $\phi: k \rightarrow(a)$ be an $A$-isomorphism. Then it is perfectly straightforward to check that $\phi \Sigma=\{\phi(U): U \in \Sigma\}$ is a neighborhood basis for 0 generating a proper topology on $A$. (Note that $\phi(U) \subset(a) \subset A$.) The proof is complete.

\section{REFERENCES}

1. J. O. Kiltinen, Existence, extension, and characterization of ring and field topologies, Thesis, Duke University, 1967.

2. - Inductive ring topologies, Trans. Amer. Math. Soc. 134 (1968), 149-169.

3. M. Nagata, Local rings, Interscience, New York, 1962.

4. O. Zariski and P. Samuel, Commutative algebra, Vol. I, Van Nostrand, Princeton, N. J., 1958.

UNIVERSITY OF MINNESOTA 